\title{
Diálogos y reflexiones sobre la etnomusicología en Chile: reunión fundacional del Comité Chileno del Consejo Internacional para la Música Tradicional (ICTM-Chile)
}

El 18 de octubre de 2019 se realizó la jornada de constitución del Comité Nacional Chileno del Consejo Internacional para la Música Tradicional (International Council for Traditional Music, o ICTM) en el auditorio del Centro de Extensión (CENTEX) del Ministerio de las Culturas, las Artes y el Patrimonio (MINCAP), en Valparaíso, Chile. El ICTM es una organización no gubernamental fundada en 1947 en relaciones formales de consulta con la UNESCO, dedicada principalmente a la investigación, práctica, documentación, conservación y difusión de la música, la danza y los bailes tradicionales. A lo largo de la última década, esta organización ha realizado varias iniciativas a favor de la etnomusicología en América Latina culminando en la formación del "Grupo de Estudios sobre Música y Danza en Latinoamérica y el Caribe" (ICTM LATCAR). Dicho grupo celebró su primer simposio en Uruguay durante el 2018, y su segunda versión en marzo del 2020 en México.

La actividad reportada tuvo como objetivo principal la instalación de un debate en relación con la identidad disciplinaria de la etnomusicología en América Latina y particularmente en Chile, así como la proyección de futuras iniciativas al respecto. Desde esta perspectiva, fue fundamental iniciar la discusión conociendo las preocupaciones sociopolíticas, los mecanismos con los que los etnomusicólogos chilenos se relacionan con el sonido y la expresión musical, acerca de sus paradigmas epistemológicos-teóricos y métodos de investigación en común, entre otros aspectos. A partir de esto, la intención respecto de la fundación de esta comunidad de investigadores es explorar y estimular el debate en Chile, mediante una reflexión colectiva respecto de las músicas tanto en términos históricos como socioculturales. La etnomusicología como disciplina va en aumento en Chile, con diversos enfoques que incluyen el estudio de músicas tradicionales, de músicas de los pueblos originarios, de la música popular por medio de la etnografía, y de los procesos y debates en torno a la preservación y el desarrollo de nuestros diversos patrimonios musicales. De esta manera, la jornada se desarrolló de la siguiente forma:

\section{Sesión de la mañana}

\section{9:30 Recepción}

10:00 Palabras inaugurales -Jacob Rekedal (Universidad Alberto Hurtado) y Agustín Ruiz (MINCAP)

10:15 Comentarios de Franco Daponte acerca de gestión e investigación participativa

10:30 Conferencia Internacional: "Patrimonio cultural, desarrollo social y bienestar comunitario", Dan Bendrups (La Trobe University, Australia)

11:00 Presentación de libro: Si tú nos prestas la vida, Daniel González de Etnomedia

12:00 Sesión fundacional del comité chileno de ICTM

Sesión de la tarde

15:00 Simposio

Ponencia 1: "Rosa o la cantautora desdoblada: Voz, memoria y proyección del rol tradicional de una cantora aymara en Iquique", Rosario Mena (Universidad Alberto Hurtado) 
Ponencia 2: "El cachimbo. Revitalización y salvaguardia de un patrimonio cultural inmaterial en Chile", Jean Franco Daponte (Universidad de Tarapacá)

16:00 Mesa temática: "Desempeño de la investigación musical en la esfera de la gestión pública", moderador: Agustín Ruiz (MINCAP)

17:00 Cierre.

El evento se inició con las palabras inaugurales de Agustín Ruiz y Jacob Rekedal, quienes contextualizaron el objetivo del proceso de constitución del Comité Nacional. Las reflexiones se iniciaron con la instalación de las tensiones existentes entre estilos y motivos de investigaciones basadas en el sector público versus academia. Se espera que ICTM contribuya a la instalación de espacios de diálogo al respecto, y por lo mismo, se realizó esta reunión en el mismo MINCAP. Por otra parte, la incorporación de etnomusicólogos chilenos al ICTM posibilitará un diálogo más global, permitiendo establecer redes investigativas que nutran los trabajos musicológicos de investigadores nacionales, especialmente considerando la relación entre ICTM y UNESCO. He ahí un aspecto fundamental de este comité: proveer una institucionalidad para la emergente masa crítica asociada a la investigación etnomusicológica a nivel nacional, desde una perspectiva descentralizada.

Para finalizar las palabras iniciales, Rekedal dio lectura a una carta emitida por la directiva de la Sociedad Chilena de Musicología. En aquella comunicación se dio la bienvenida al Comité Chileno, reafirmando la relevancia de abrir espacios de diálogo, por cuanto ambas organizaciones comparten diversos elementos en común y, en paralelo, contribuyen al desarrollo de un círculo virtuoso que posibilitará el establecimiento de diversos trabajos académicos. Se concluyó que la relación entre el Comité Nacional de ICTM y la Sociedad Chilena de Musicología contribuirá al establecimiento de un círculo virtuoso que permita seguir afirmando la masa crítica para el desarrollo de la etnomusicología chilena.

La primera presentación estuvo a cargo del Dr. Franco Daponte Araya, investigador de la Universidad de Tarapacá, quien habló de su experiencia en relación con la gestión patrimonial y la investigación participativa e interdisciplinar. Compartió parte de su trabajo que derivó en la designación del baile de cachimbo como patrimonio cultural inmaterial de la región de Tarapacá. Daponte mencionó las dificultades, así como las ventajas que se presentan en el desarrollo de un trabajo que integra a las comunidades directamente en la labor de investigación. Debido a que Daponte es oriundo de la zona y cultor de esta práctica, señaló que su calidad de insider fue un aspecto clave para el establecimiento de una relación recíproca con los demás cultores. Las actividades desarrolladas incluyeron un conversatorio con las comunidades, que le permitió compartir e interpretar hallazgos con los cultores, así posibilitando una reflexión dialógica y la articulación de ciertos conceptos tradicionales. Por ejemplo, se habló acerca del "Aire del cachimbo", lo que resultó ser un elemento musical determinado por un musema ligado a un esquema rítmico específico, cuyo significado es socialmente identificado. La experiencia investigativa permitió la instalación de procesos interdisciplinarios y transdisciplinarios que, en el caso particular de la región de Tarapacá, surgió a partir de un enfoque etnohistórico. Dicho enfoque permitió entrelazar la geografía, historia, musicología y genealogía, entre otras disciplinas, generando representaciones científicas diversas. El investigador concluyó que las experiencias dialógicas e interdisciplinarias permitieron enriquecer las conclusiones de su investigación, lo que reafirma la necesidad de generar espacios de diálogo, y de participación de comunidades y cultores en trabajos etnomusicológicos.

De manera posterior, se realizó la conferencia internacional a cargo del Dr. Dan Bendrups, de la Universidad La Trobe, Australia, titulada "Futuros Sonoros: patrimonio cultural, desarrollo social y el bienestar comunitario". La comunicación se inició con un análisis de cómo la etnomusicología chilena se ha desarrollado, específicamente a partir del estudio de la música de Rapa Nui. Se realizó una revisión de los investigadores que han estudiado la música de la isla, profundizando en el caso de Ramón Campbell, quien documentó más de un centenar de canciones tradicionales. Dicho autor, señala Bendrups, es uno de los primeros investigadores explícitamente afiliado con la disciplina de etnomusicología. A pesar de la ausencia de una base teórica, el concepto de etnomusicología en Chile se instaló -al menos como neologismo-, en una época paralela a la instalación del campo disciplinario por los autores tradicionales (Alan Merriam, entre otros, desde mediados del siglo XX). La presentación abordó tres estudios de caso: una investigación de Rapa Nui, una de la migración chilena en Australia y una experiencia de etnomusicología aplicada en Indonesia. El autor se pregunta: ¿cómo entender el patrimonio cultural?, ¿cuáles son las relaciones posibles entre la música y el desarrollo social?, ¿cuáles son los futuros etnomusicológicos posibles? 
El primer caso expuesto correspondió al Proyecto Felbermayer, donde Bendrups colaboró con miembros del Museo Fonck en Viña del Mar, región de Valparaíso, el que se desarrolló en 2013 a partir de una colección fotográfica y grabaciones de audio encontradas en su repositorio. Se encontró una cinta, hasta el momento desconocida, que contenía cuarenta y cinco pistas con extractos de la misa Rapa Nui (himene pure), cantos profanos (himene api), cantos de origen tahitiano (himene tahiti), recitaciones de kai kai, recitaciones de a'amu tuai y ejemplos de rima apu (manera tradicional de tocar guitarra). Si bien lo que se encontró no representaba información nueva -por cuanto era información analizada en estudios previos-, sí se pudo constatar la presencia de diversas canciones de origen tahitiano. El estudio concluye planteando las siguientes preguntas: ¿el patrimonio cultural puede existir en un artefacto, de raíz foránea?, ¿qué nos dice el artefacto?, ¿Rapa Nui tuvo una condición más globalizada que el Chile de aquel entonces?

El segundo estudio reportó los resultados de un proceso indagatorio acerca de la salsa en Melbourne, Australia, y su vínculo con la migración chilena. Bendrups realizó un pequeño recorrido histórico de la presencia de músicos chilenos en los diversos procesos migratorios hacia Australia. Mencionó que en la década de 1960 se produjo la primera oleada de inmigrantes chilenos a Australia, y luego otra en los años setenta, esta vez motivada por el exilio político. Pero no es hasta la década de 1980 donde se pueden identificar agrupaciones musicales constituidas por chilenos, argentinos, salvadoreños, entre otros, muchas de ellas ligadas al movimiento laborista. A finales de esta década, particularmente en 1987 y relacionada al boom del grupo Gipsy Kings, se evidencia el surgimiento del interés por la música de América Latina. Ya en 1990 se observan entre ocho y doce agrupaciones tocando de manera regular (aproximadamente cuatro veces por semana). A inicios del siglo XXI, una nueva generación de chileno-australianos consideran la música como una fuente de empleo viable, en donde se pueden identificar agrupaciones de diversos estilos, siendo la salsa de oro el más significativo. La razón se debe, principalmente, a que este estilo es temáticamente inclusivo y con tintes políticos, destacándose el catálogo musical de Colón/Blades. La participación de las comunidades es significativa: permite potenciar un sentido de pertenencia, es un acto de celebración de una identidad cultural, una forma de protesta encubierta y, más tarde, una posibilidad de empleo.

El tercer y último caso corresponde a una investigación aplicada, acerca de las relaciones entre salud, bienestar y música, que se realizó en una zona rural de la isla de Java, en Indonesia. A partir de la premisa que indica que la música puede influenciar en ciertos determinantes sociales de salud, se revisaron variados estudios asociados al campo de la etnomusicología médica. Sobre la base de dicha reflexión, Bendrups nos recordó de la importancia del siguiente cuestionamiento: ¿Es posible movilizar la etnomusicología en una forma aplicada, como una forma de establecer soluciones en pos de mejorar el bienestar social?

Como respuesta, expuso que ciertas comunidades padecían de variados y graves problemas de salud, principalmente enfermedades intestinales y parasitarias. En todos los casos, Bendrups mencionó que las soluciones estaban asociadas a la higiene, por lo que la educación concerniente a este tema parecía ser la alternativa más eficiente. Sobre la base de esto, se preguntaron, ¿cómo ofrecer educación en un contexto de baja alfabetización y con diversos idiomas, además de poco contacto con servicios médicos? La determinación del investigador fue realizar un trabajo audiovisual, que incorporara elementos tradicionales de la música de Java, con contenidos asociados a la higiene. De esta manera surge Rama and The Worm (Rama y el gusano), un corto que utiliza la técnica tradicional wayang kulit (teatro/ títeres de sombras) que cuenta la historia del príncipe Rama (personaje recurrente en este género) y un gusano cuya figura es basada en una fotografía microscópica de un parásito intestinal. Dicho material audiovisual contó con una narración ideada por Bendrups y el Dr. Joko Susilo. Para la música, se utilizó una combinación entre gamelán e instrumentos asociados al rock (vientos, percusión y varios efectos electrónicos). Finalmente se realizó un DVD que fue entregado a las comunidades ${ }^{1}$. Bendrups concluyó diciendo que la intervención fue exitosa, ya que permitió generar un proceso de enseñanza que posibilitó una disminución en los procesos infecciosos derivados de las problemáticas de higiene.

A continuación se dio paso a la presentación del libro Si tú nos prestas la vida: La devoción popular de los bailes chinos y sus fiestas, de Rafael Contreras y Daniel González. Durante la exposición, que estuvo a cargo de González, se mencionó que esta publicación es una especie de segundo volumen de

1 El producto realizado se encuentra -en su versión en inglés- disponible en: https://youtu.be/ AafNmmMlrvQ [acceso: 18 de octubre de 2019]. 
libro Será hasta la vuelta de año (2015), que muestra documentos y reflexiones teóricas que surgieron a partir de ese trabajo que se inició en 2002. La publicación de los dos libros se dio en el contexto de la postulación e inscripción, en 2014, de los bailes chinos en la Lista Representativa del Patrimonio Cultural Inmaterial de la humanidad. Los libros tienen un carácter casi enciclopédico, y dan cuenta de los diversos elementos de estas prácticas musicales, así como de los productos generados al respecto, como, por ejemplo, documentales². González comenta que la investigación acerca de los bailes chinos resulta atractiva, y por esta razón el texto busca indagar en las dimensiones internas y externas de los bailes, evitando reiterar la crónica como descripción externa, para indagar en problemáticas más complejas. A partir de esto, surgen dos conceptos: la heterogeneidad y la hegemonía, que fueron trabajados en dos dimensiones:

1) Dimensión del trabajo: la relación entre los bailes chinos y los trabajadores. Esto permitió explicar la fiesta desde la perspectiva del trabajo y su relación con los trabajadores, reflexionando respecto de la elaboración de los bailes como un trabajo en sí mismo. Desde esta perspectiva, la palabra chino suele relacionarse con los contextos de trabajo extenuantes. Se observa la generación de microcolectivos, lo que evidenciara un rol social relevante de los bailes chinos, asociado a una respuesta a ciertas prácticas de explotación que datan de la Colonia.

2) Dimensión de lo simbólico, la expresión y la estética: se indagó la relación de las diferencias estéticas, visuales y simbólicas que permiten comprender las razones por las que surgen los bailes. Esto permite generar un complejo mapa de interrelaciones expresivas asociadas a los contenidos líricos, orales y visuales, que por su parte permiten establecer mecanismos de expresión. En este sentido, el libro posee una recopilación de aproximadamente ciento veinte años de poesía.

Desde el punto de vista metodológico, el libro aborda el estudio de las historias de vida como estrategia que permite deconstruir las percepciones convencionales en relación con los bailes chinos. Es la subjetividad de los cultores que permite establecer mecanismos de diálogos con los investigadores. Se concluye que el concepto de chinos tiene una problemática inherente a su condición, por cuanto su definición parece no ser -únicamente- la forma en la que se encuentra delimitada por los medios hegemónicos. Durante la investigación, se invitó a una reflexión conjunta con los cultores en relación con las formas en las que dichos conceptos han mutado al interior de las prácticas.

La sesión de la mañana culminó con la reunión fundacional del comité chileno del ICTM. En este contexto, Jacob Rekedal en su función de Chile Liaison Officer del ICTM, resumió lo que conlleva la instalación de un comité nacional, y las ventajas que implica estar asociado a esta organización internacional. Luego se dio paso a la elección de la directiva para formalizar el proceso fundacional; Rekedal fue elegido presidente del comité, Javier Silva-Zurita secretario, e Ignacio Soto Silva tesorero. Se acordó realizar una nueva reunión a principios de 2020, con el fin de convocar a nuevos miembros y programar futuras actividades. Una vez culminada la reunión, se realizó un receso, retomando en la jornada de la tarde con un breve simposio donde se presentaron Rosario Mena y Franco Daponte.

Rosario Mena, estudiante del programa de Magíster en Musicología Latinoamericana de la Universidad Alberto Hurtado, partió con su exposición titulada "Rosa o la cantora desdoblada: Voz, memoria y proyección del rol tradicional de una cantora aymara en Iquique", la que trató del canto harawi y su relevancia en las distintas etapas de la vida de la mujer andina. Mena señaló que esta práctica es un elemento central en ciertos rituales, con relevancia especial en los procesos educacionales y de sanación. Agregó que las mujeres han utilizado el canto como un mecanismo de conformación de identidad tradicional, citando el caso de Rosa Quispe quien se desempeña como cantora y educadora tradicional, dedicada a la enseñanza de la lengua aymara. Mena explicó que, desde niña, Quispe practicó los cantos tradicionales a través de las enseñanzas de su madre y su abuela, y que en la actualidad ha llegado a grabar discos para ser utilizados en establecimientos educacionales de Iquique. Mena puntualizó el uso de ciertas marcas identitarias que son características de las cantoras andinas, en complemento con ciertos elementos propios asociados al paisaje sonoro andino, como, por ejemplo, la presencia del registro agudo, que según Quispe se asocia a la espiritualidad.

2 www.baileschinos.cl [acceso: 18 de octubre de 2019]. 
Mena concluyó que el canto es una forma de postmemoria, que reelabora el trauma del desarraigo y la colonización, formando un vínculo con la naturaleza que se expresa en temáticas asociadas a animales y la utilización de elementos del paisaje sonoro. Finalmente, los modos de apropiación de la cantora andina evidencian un proceso complejo de interrelación entre la visión tradicional de la cantora tradicional y los estereotipos occidentales de la mujer.

La última ponencia de esta sección, titulada "El Cachimbo: Revitalización y salvaguardia de un Patrimonio Cultural Inmaterial en Chile”, estuvo a cargo del Dr. Franco Daponte, quien profundizó en las temáticas asociadas a esta danza y su proceso de patrimonialización. En este sentido, el expositor reporta las problemáticas que surgen a partir de la presencia de un cachimbo urbano que se encuentra al margen de las manifestaciones incluidas por el Ministerio de las Culturas, las Artes y el Patrimonio (MINCAP) en su catálogo de patrimonio inmaterial. Para esto, realizó una caracterización geográfica y estilística de esta danza, puntualizando las diferencias que surgieron durante el siglo XX a propósito de su localización geográfica, lo que implicó que, en las distintas locaciones, en Pica, Tarapacá, Mamiña y Matilla, se ejecutara de formas diversas. A partir de esto, Daponte realizó un recuento histórico del desarrollo del cachimbo desde su origen peruano republicano hasta el presente, y cómo esta práctica musical es constitutiva de patrimonio significativo en su contexto, y también un mecanismo de resistencia.

Posteriormente, profundizó en las características socioculturales e históricas del cachimbo en el contexto urbano, explicando que su presencia en Iquique ha significado un mecanismo de construcción de identidad, reconfigurando un imaginario del "iquiqueño del interior" que genera tensiones con los cultores más antiguos quienes no la reconocen como tradicional. Finalmente, sintetizó acerca de diversas formas de salvaguardia articuladas por los habitantes de las localidades del interior, y cómo el cachimbo urbano establece un nuevo escenario para el estudio de esta manifestación.

La sección final de la jornada de trabajo y reflexión estuvo a cargo de Agustín Ruiz, quien moderó una mesa de discusión titulada "Desempeño de la investigación musical en la esfera de la gestión pública”. Dicha mesa contó con la participación de Patricio Díaz y Luis Vildósola, ambos del MINCAP, quienes contribuyeron a la realización de un diálogo colectivo respecto del rol de la etnomusicología y del etnomusicólogo en el contexto de la gestión pública. Se revisaron variados proyectos del MINCAP, en donde la presencia de un enfoque etnomusicológico contribuyó significativamente al desarrollo de elementos que han permitido ciertos posicionamientos disciplinarios. Sin perjuicio de lo anterior, se concluyó que este contexto es propicio para la generación de planes formativos en las universidades, que posibiliten la inserción laboral de los etnomusicólogos en el sistema público. La visión de un profesional de esta disciplina posiblemente contribuya de manera significativa a la instalación de procesos que consideren las particularidades del material musical y de los saberes tradicionales.

En el cierre de la actividad, el presidente del Comité Chileno reflexionó pertinente a la relevancia de instalar de forma dialógica y sistemática, procesos de conversación y divulgación en relación con el rol de la etnomusicología en el estudio de las músicas tradicionales. Sin lugar a duda, la conformación de equipos investigativos y la planificación de estudios etnomusicológicos que permitan realizar acompañamientos y aplicar ciertos aspectos metodológicos de la disciplina, serán fundamentales para la generación de un círculo virtuoso entre las distintas sociedades científicas, así como la Sociedad Chilena de Musicología, el ICTM, y con otras comunidades de músicos e investigadores.

Ignacio Soto Silva Departamento de Humanidades y Artes, Universidad de Los Lagos, Chile. Tesorero Comité Chileno ICTM ignacio.soto@ulagos.cl

Jacob Rekedal

Instituto de Música, Universidad Alberto Hurtado, Chile Presidente Comité Chileno ICTM jrekedal@uahurtado.cl

Javier Silva-Zurita

INTE, Universidad Arturo Prat, Chile. Secretario Comité Chileno ICTM javier.silva.zurita@gmail.com 